\title{
Comparative analysis of plant carbohydrate active enZymes and their role in xylogenesis
}

\author{
Desre Pinard ${ }^{1}$, Eshchar Mizrachi ${ }^{1}$, Charles A Hefer ${ }^{2}$, Anna R Kersting $^{3}$, Fourie Joubert ${ }^{2}$, Carl J Douglas ${ }^{4}$, \\ Shawn D Mansfield ${ }^{5}$ and Alexander A Myburg ${ }^{1 *+}$
}

\begin{abstract}
Background: Carbohydrate metabolism is a key feature of vascular plant architecture, and is of particular importance in large woody species, where lignocellulosic biomass is responsible for bearing the bulk of the stem and crown. Since Carbohydrate Active enZymes (CAZymes) in plants are responsible for the synthesis, modification and degradation of carbohydrate biopolymers, the differences in gene copy number and regulation between woody and herbaceous species have been highlighted previously. There are still many unanswered questions about the role of CAZymes in land plant evolution and the formation of wood, a strong carbohydrate sink.

Results: Here, twenty-two publically available plant genomes were used to characterize the frequency, diversity and complexity of CAZymes in plants. We find that a conserved suite of CAZymes is a feature of land plant evolution, with similar diversity and complexity regardless of growth habit and form. In addition, we compared the diversity and levels of CAZyme gene expression during wood formation in trees using mRNA-seq data from two distantly related angiosperm tree species Eucalyptus grandis and Populus trichocarpa, highlighting the major CAZyme classes involved in xylogenesis and lignocellulosic biomass production.
\end{abstract}

Conclusions: CAZyme domain ratio across embryophytes is maintained, and the diversity of CAZyme domains is similar in all land plants, regardless of woody habit. The stoichiometric conservation of gene expression in woody and non-woody tissues of Eucalyptus and Populus are indicative of gene balance preservation.

Keywords: Carbohydrate active enzymes, Comparative genomics, Transcriptomics, Eucalyptus grandis, Protein domains, Wood formation, Populus trichocarpa

\section{Background}

Carbohydrate metabolism in plants is responsible for a diverse array of developmental processes, including energy metabolism, signaling, defense, cell wall (CW) structure [1], and carbohydrate-related post-translational modifications [2]. Carbohydrate biopolymers in the secondary cell walls (SCWs) of fiber cells form the bulk of woody biomass, a valuable natural resource with a variety of industrial applications, including pulp and paper, and potential biofuel production [3,4]. The vessel and fiber cells in angiosperm wood have significant amounts of cellulose, hemicellulose and lignin in their SCWs

\footnotetext{
* Correspondence: zander.myburg@up.ac.za

${ }^{\dagger}$ Equal contributors

'Department of Genetics, Forestry and Agricultural Biotechnology Institute (FABI), University of Pretoria, Private bag X20 Hatfield, Pretoria 0028, South Africa

Full list of author information is available at the end of the article
}

[5,6]. Cellulose and hemicelluloses are synthesized, modified, and degraded by Carbohydrate-Active enZymes (CAZymes), a group comprising of modular protein domains that are ubiquitous across all living organisms [7-9]. CAZymes have been classified into four classes of enzymatic domains, namely glycosyl transferases (GTs), glycoside hydrolases (GHs), polysaccharide lyases (PLs) and carbohydrate esterases (CEs) $[9,10]$. In addition, a non-enzymatic class exists, the carbohydratebinding modules (CBMs) [11,12]. Currently, the five CAZyme classes are collected and organized into families based on amino acid sequence similarity in the CAZy database $[8,13]$.

GTs catalyze glycosyl bonds between a donor sugar substrate and another molecule, typically another sugar [14]. Along with defense, signaling and storage carbohydrate biosynthesis, plant GTs are responsible for the production of cellulose (GT2 domain family- Cellulose 
synthase A (CESA) gene superfamily) [15] and hemicelluloses (GT2, GT8, 43, 47, and 61 families, among others) [16-21]. GH domains hydrolyze the glycosyl bonds between sugars in carbohydrate biopolymers [9]. They play an important role in the modification of biopolymers to be introduced into the CW, as well as abscission and dehiscence [22]. PLs are implicated in non-hydrolytic cleavage of activated glycosidic bonds in pectin modification and degradation $[23,24]$. CEs deacetylate polysaccharide side-chains, and are thought to modify the cross-linking of hemicellulose with lignin $[25,26]$. CBMs allow for specific binding to different carbohydrate biopolymers, facilitating precise biopolymer modifications by enzymatic domains as they are added to the CW $[27,28]$. Due to their ability to disrupt the SCW network by binding to $\mathrm{CW}$ polymers, CBMs have been used in industry to increase the efficiency of $\mathrm{CW}$ degradation during the pulping process [29].

Previous studies have found higher frequency and diversity of CAZyme genes in the genome of $P$. trichocarpa than that of $A$. thaliana [30], which in 2001, had the most annotated CAZymes in its genome compared to sequenced fungal and bacterial species [11]. Furthermore, the CAZymes expressed in wood-forming tissues of $P$. trichocarpa, specifically those involved in cellulose and hemicellulose biosynthesis, are more abundant and diverse than those in non-wood forming tissue such as young leaves [30]. Based on these findings, the authors noted the importance of CAZymes to woody characteristics.

Protein domains, as the functional and evolutionary building blocks of plant proteins, are informative of the functional capacity of the genome [31,32]. A recently published database of CAZyme domains, dbCAN $[33,34]$, can be employed to identify the frequency and diversity of CAZyme domains in plant genomes available on Phytozome [35]. dbCAN utilizes Hidden Markov Models (HMMs), based on the seminal CAZyme family sequence data available [13], to accurately and reproducibly identify CAZyme domains [33]. Using the dbCAN database, protein-coding genes containing CAZyme domains in twenty-two plant species can be compared to analyze their CAZyme domain repertoire.

Fundamental questions about the evolution of woody plant secondary growth still exist [36], and with the availability of the genome of a second hardwood species, that of $E$. grandis, along with mRNAseq data for $E$. grandis and $P$. trichocarpa [37], some of these questions can be addressed. We aimed to characterize the CAZyme domain frequency and diversity in plant species, and their expression levels in P. trichocarpa and $E$. grandis woody and non-woody tissues. In this way, we could identify the common expressed CAZyme repertoires involved in carbohydrate metabolism in woodforming tissues of two evolutionary divergent tree genera. Specifically, we asked: Does the frequency and diversity of CAZyme domains between plants reflect their evolution and developmental complexity? Is the expression of CAZyme domains related to wood formation in E. grandis and P. trichocarpa? We hypothesized that the genomes of woody trees would exhibit gains or expansions of CAZyme domain containing genes that contribute to carbohydrate biopolymer formation and deposition in wood formation. Further hypotheses were that the expression investment of CAZyme domaincontaining genes abundant in the developing xylem would be higher than in non-xylogenic tissue as a reflection of focused carbohydrate metabolism in this sink tissue. This study is the first systematic analysis of genome-wide and expressed CAZyme domains across a diversity of plant species, with a focus on CAZyme utilization for the production of lignocellulosic biomass.

\section{Results}

Genome-wide analysis of CAZyme classes in plants

To gain insight into the evolution of CAZyme genes across key land plant evolutionary lineages, we compared the domain content of twenty-two plant species that have been annotated in dbCAN from Phytozome by examining the number of genes containing CAZyme domains, and the frequency of CAZyme domains in these genes within each plant genome (Table 1). The absolute frequency of genes from each CAZyme class per genome shows that seed producing plants (except Carica papaya-1,341 CAZyme domains) have more CAZyme containing genes and CAZyme domains than non-seed plants such as the bryophyte Physcomitrella patens (1,519 CAZyme domains) and the lycophyte Selaginella moellendorffii (1,476 CAZyme domains), and almost double that of the green algal species Volvox carteri and Chlamydomonas reinhardtii (654 and 731 CAZyme domains, respectively) (Figure 1a, Table 1). However, the absolute frequencies of these genes in angiosperms can be misleading, as some plant genomes have undergone one or more whole genome duplications (WGD) and experienced extensive gene loss in the past [38-40]. The absolute gene frequency may reflect the age of the genome since the last WGD and the rate of gene loss in the lineage, as well as other mechanisms such as gene retention after neo/sub-functionalization, or tandem gene duplication $[38,39,41]$.

Although the absolute frequencies of CAZy domains vary between plant genomes, we found that the proportions of the five functional CAZymes classes (GT, GH, CE, PL, CBM) are remarkably similar among species (Figure $1 \mathrm{a}, \mathrm{b}$ ). A co-efficient of variance analysis was performed to determine if the ratios of CAZyme classes among monocots, eudicots, lycophytes and bryophytes, and green algae varied significantly. For all CAZyme 
Table 1 Genome- wide CAZyme gene and domain content for twenty-two plant species

\begin{tabular}{|c|c|c|c|c|c|c|c|c|c|c|c|}
\hline Organism* & $\begin{array}{l}\text { Genome Size } \\
\text { (Mbp) }\end{array}$ & \#Genes & $\begin{array}{l}\text { \#CAZyme } \\
\text { genes }\end{array}$ & $\begin{array}{l}\text { \%CAZyme } \\
\text { genes }\end{array}$ & $\begin{array}{l}\text { \#CAZyme } \\
\text { domains }\end{array}$ & $\begin{array}{l}\# \\
\text { GTs }\end{array}$ & $\begin{array}{l}\# \\
\text { GHs }\end{array}$ & $\begin{array}{l}\# \\
\text { PLs }\end{array}$ & $\begin{array}{l}\# \\
\text { CEs }\end{array}$ & $\begin{array}{l}\# \\
\text { CBMs }\end{array}$ & Reference \\
\hline Volvox carteri (VolCa) & 138 & 14,520 & 490 & 3.37 & 645 & 283 & 85 & 4 & 65 & 102 & {$[67]$} \\
\hline $\begin{array}{l}\text { Chlamydomonas } \\
\text { reinhardtii (ChIRe) }\end{array}$ & 121 & 15,143 & 574 & 3.79 & 741 & 367 & 85 & 4 & 76 & 87 & {$[68]$} \\
\hline $\begin{array}{l}\text { Physcomitrella patens } \\
\text { (PhyPa) }\end{array}$ & 480 & 35,938 & 1,236 & 3.44 & 1,519 & 664 & 392 & 41 & 250 & 172 & [69] \\
\hline $\begin{array}{l}\text { Selaginella moellendorffii } \\
\text { (SelMo) }\end{array}$ & 212 & 22,285 & 1,224 & 5.49 & 1,476 & 637 & 370 & 27 & 281 & 161 & [70] \\
\hline $\begin{array}{l}\text { Brachypodium distachyon } \\
\text { (BraDi) }\end{array}$ & 272 & 25,532 & 1,418 & 5.55 & 1,723 & 764 & 394 & 20 & 334 & 211 & [71] \\
\hline Oryza sativa (OrySa) & 420 & 42,109 & 1,724 & 4.09 & 2,040 & 891 & 498 & 29 & 389 & 233 & [72] \\
\hline Zea mays (ZeaMa) & 2,300 & 30,579 & 1,920 & 6.28 & 2,256 & 1,026 & 578 & 22 & 394 & 236 & [73] \\
\hline Sorghum bicolor (SorBi) & 730 & 34,496 & 1,751 & 5.08 & 1,431 & 784 & 474 & 23 & 288 & 171 & [74] \\
\hline $\begin{array}{l}\text { Aquilegia coerulea } \\
\text { (AquCo) }\end{array}$ & 302 & 24,823 & 1,554 & 6.26 & 1,464 & 657 & 471 & 32 & 360 & 141 & [75] \\
\hline Mimulus guttatus (MimGu) & 312 & 26,718 & 1,671 & 6.25 & 1,992 & 680 & 503 & 43 & 363 & 201 & [76] \\
\hline Vitis vinifera $(\mathrm{VitVi})$ & 490 & 30,434 & 1,424 & 4.68 & 1,710 & 664 & 525 & 39 & 305 & 177 & [77] \\
\hline Eucalyptus grandis (EucGr) & 641 & 36,376 & 2,542 & 6.99 & 3,334 & 1,233 & 823 & 54 & 534 & 360 & {$[37]$} \\
\hline Citrus clementina (CitCl) & 301 & 24,533 & 1,971 & 8.03 & 2,328 & 862 & 572 & 41 & 413 & 205 & [78] \\
\hline Citrus sinensis (CitSi) & 319 & 25,376 & 2,439 & 9.61 & 2,927 & 1,049 & 735 & 52 & 498 & 267 & [78] \\
\hline Carica papaya (CarPa) & 372 & 27,873 & 1,131 & 4.06 & 1,341 & 466 & 380 & 25 & 209 & 124 & [79] \\
\hline $\begin{array}{l}\text { Arabidopsis thaliana } \\
\text { (AraTh) }\end{array}$ & 119 & 27,400 & 1,505 & 5.49 & 1,787 & 697 & 483 & 49 & 341 & 217 & {$[80]$} \\
\hline Prunus persica (PruPe) & 227 & 27,852 & 1,591 & 5.71 & 1,843 & 654 & 491 & 34 & 337 & 180 & [81] \\
\hline Cucumis sativus (CucSa) & 243 & 26,682 & 2,157 & 8.08 & 2,157 & 779 & 555 & 36 & 355 & 184 & [82] \\
\hline Glycine max (GlyMa) & 975 & 54,175 & 2,839 & 5.24 & 3,429 & 1,412 & 917 & 69 & 645 & 386 & [83] \\
\hline $\begin{array}{l}\text { Populus trichocarpa } \\
\text { (PopTr) }\end{array}$ & 422 & 41,335 & 2,252 & 5.45 & 2,677 & 1,057 & 713 & 55 & 479 & 373 & [84] \\
\hline Ricinus communis (RicCo) & 350 & 31,237 & 1,540 & 4.93 & 1,864 & 605 & 486 & 36 & 328 & 186 & {$[85]$} \\
\hline $\begin{array}{l}\text { Manihot esculenta } \\
\text { (ManEs) }\end{array}$ & 533 & 30,666 & 1,957 & 6.38 & 2,365 & 825 & 616 & 42 & 377 & 239 & {$[86]$} \\
\hline
\end{tabular}

*The first column shows the plants analysed with the abbreviations used in this study.

domain classes except PLs, the difference in the frequency ratios among the land plant (excluding the green algae) classes was negligible (Additional file 1: Table S1). In land plants, GTs comprised roughly $40 \%$ of the CAZyme domain content in the genome, with GHs having a relative frequency of $30 \%$. CEs, PLs and CBMs have relative frequencies of 18,2 and 10 percent, respectively. In contrast, the green algae have frequency ratios of approximately $57 \%$ for GTs, $14.5 \%$ for GHs, $0.6 \%$ for PLs, $10 \%$ for CEs and $17 \%$ for CBMs.

\section{Genome-wide comparison of CAZy domain diversity and complexity}

Next, we asked what the diversity of CAZyme families within each class is between plant species. Here the objectives were to analyze the CAZyme domain families within each broader functional class present in each genome to determine whether the presence of unique domain families contributed to the organismal complexity of vascular and/or seed producing plants. All twentytwo species from the previous analysis were analyzed to determine the diversity of the domain families present in each species (Additional file 2, Table 1). There are 231 different CAZyme domains present across the plant lineages analyzed ( $72 \mathrm{GT}, 92 \mathrm{GH}, 13 \mathrm{PL}, 16 \mathrm{CE}$ and 38 CBM families). The frequency of all families across the 22 species displayed a bimodal distribution $\left(R^{2}=0.68\right)$, either present in all species, ubiquitous to all land plants considered, or present only in one or two species (Additional file 3: Figure S1). This bimodal pattern has been observed more generally in the context of shared domain combinations among plants, and consistent with large shared domain gain events early in land plant evolution [32]. There are 74 domain families that are 


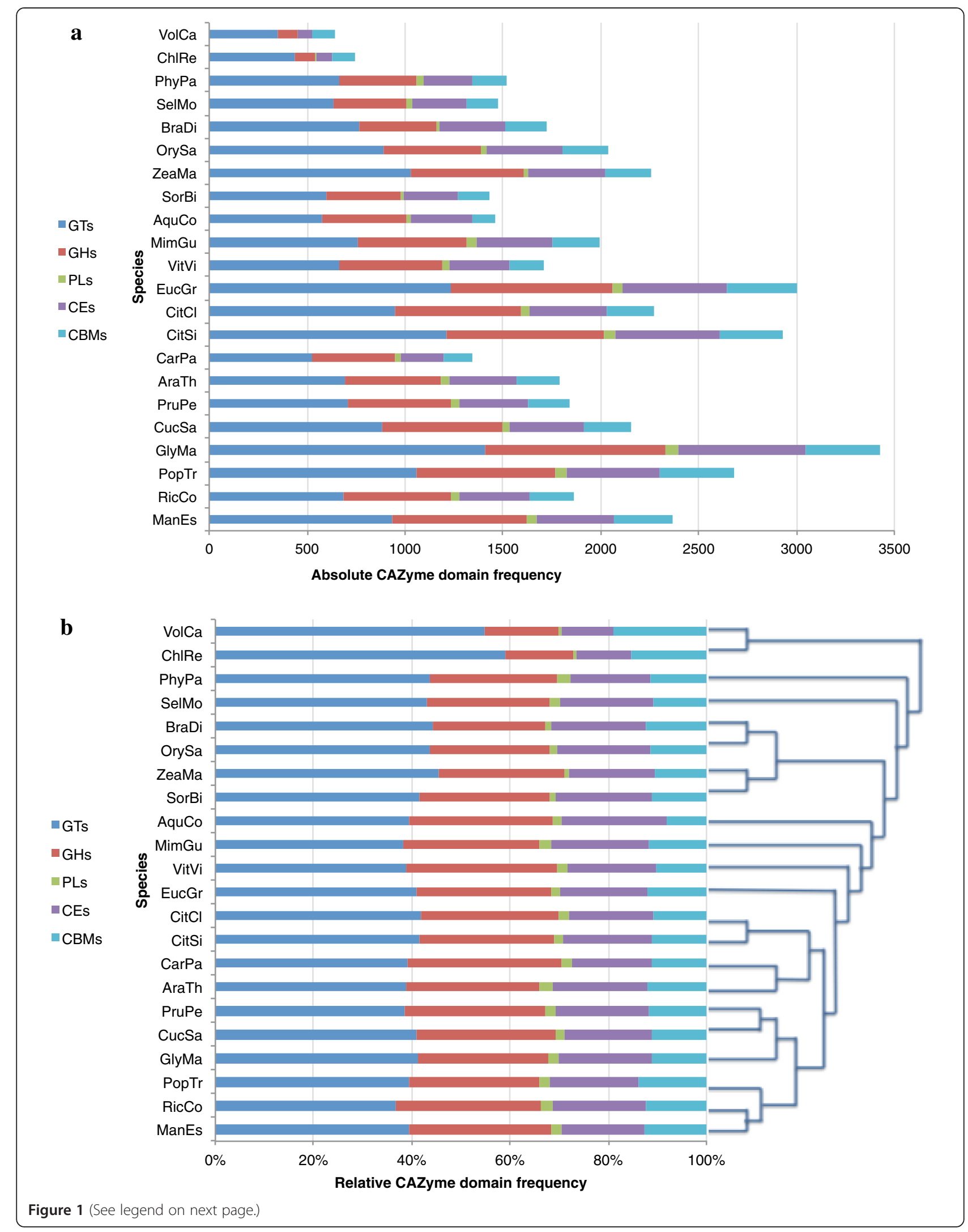


(See figure on previous page.)

Figure 1 Absolute and relative frequency of CAZyme domain class frequency across twenty-two plant species. (a) Absolute frequency of CAZyme domains in five classes across twenty-two plant species. Plant species are on the $y$-axis, and the absolute frequency of CAZyme domains within all CAZyme genes is shown on the $x$ - axis. The glycosyl transferase (GT) domain class is represented in blue, glycosyl hydrolase (GH) domain class in red, polysaccharide lyase (PL) domain class in green, carbohydrate esterase (CE) domain class in purple and carbohydrate binding module (CBM) domain class in light blue. (b) Relative frequency of CAZyme domain classes in twenty-two plant species. The relative frequency of carbohydrate active enzyme (CAZyme) domain classes in CAZyme genes, as a percentage, is shown on the $x$-axis. The species of plant is shown on the $y$-axis. For species abbreviation, refer to Table 1.

found in one or two species are representatives from the GT, GH, PL and CBM classes, with none from the CE class (Additional file 3: Figure S1, Additional file 2). 5 CBMs, $1 \mathrm{GH}$ and 5 GTs are found exclusively in the green algae, while the lycophytes and bryophytes share one GH that only occurs in those species. The rest are interspersed amongst the remaining 18 vascular seed plants. CBM17 is only found in the two citrus species, and is thought to bind to amorphous cellulose (http:// www.cazy.org/CBM17.html).

Of the 231 CAZyme domain families found across all 22 species, only 65 are common to all, and 20 occur ubiquitously across all land plants, but are absent in the green algae (Additional file 2). However, within all genomes, the frequency of these 65 conserved families account for the majority $(75 \% \pm 2 \%)$ of CAZyme domains, with the 20 land plant-specific domains accounting for an additional $12 \%-17 \%$ of CAZyme domains in these species. Only one CAZYme domain, CBM16, was observed to occur ubiquitously across seed plants, but absent in the lycophyte Selaginella moellendorffii and moss Physcomitrella patens, and the green algae Chlamydomonas rienhardtii and Volvox carteri (Additional file 2). CBM16, which is known to bind to cellulose and glucomannan, is only detected in the eighteen seed plants in the twenty-two species analyzed, although it should be noted that it is also found in Archaea and Bacteria [13]. In Eucalyptus and most other seed plant species, this CBM occurs exclusively in DUF642-domain containing proteins. The biological function of these proteins has not yet been fully resolved but they have been demonstrated to be essential for various aspects of developmental biology (see [42] for a recent review). The domain has been previously reported to be seed-plant specific, but a search revealed it is also present in conifers $[43,44]$. There were no unique domains in A. thaliana, and there were no domains that were unique to the two woody perennials, E. grandis and P. trichocarpa compared to the other plant species analyzed.

The distribution of CAZy domain-containing multidomain proteins in ten representative land plant species (eight seed plants, S. moellendorffii and P. patens) followed the power law of gene complexity and gene number ([45], Additional file 4: Figure S2). The composition of complex CAZyme proteins was considered in terms of whether they consisted solely of repeat domains, or of combinations of different domains. An average of 17\% of CAZyme proteins were found to contain repeat domains in all 10 genomes considered, of which $60 \%$ are repeats of the same CAZyme domain (Additional file 5). All CAZy domain-containing proteins that have five or more domains in all species examined contain GT41 (O-linked $\beta$-N-acetylglucosamine transferase) domains, which are involved in post-translational (O-GlcNAc) protein modification $[46,47]$.

Proteins consisting of more than one CAZyme domain show lineage specific combinations across the ten plant genomes analyzed. In the five eudicots considered, 15 CAZyme domain combinations are common to all five (Additional file 6: Figure S3). Glycine max has the most unique combinations between the eudicots at six, with $P$. trichocarpa, E. grandis and $V$. vinifera having two and $A$. thaliana having only one. Of the 28 CAZyme domain combinations that occur in E. grandis, the six that have genomic frequency greater than 10 are: CBM43-GH17, GH28-GH55, CBM18-GH19, CBM22-GH10, CE1-CE10 and CE1-CE7 (Additional file 7). Similarly in P. trichocarpa, of the 25 CAZyme domain combinations that occur more than 10 times in the genome are CBM43-GH17, CBM18-GH19 and GH28-GH55 (Additional file 7). CBMs in combination are thought to act as enhancers and mediators of the enzymatic action of their appended domains. In the E. grandis dataset, this co-operative relationship is evident in the activity of the enzymatic domain and the specificity of the attached CBM. For example, CBM 43 binds to $\beta-1,3$-glucan, and GH17 is a $\beta-1,3$-endoglucanase [13]. Similarly, CBM22 binds specifically to xylan and GH10 is a xylanase. Combinations with CBM domains are prevalent in the E. grandis genome, with CBM to enzymatic CAZyme domain combinations accounting for 11 of the 28 combinations.

\section{Expression of CAZyme domain containing genes in $E$. grandis}

The newly sequenced genome of E. grandis [37] as well as RNA-sequencing data for several tissues and organs [48] allowed a functional genomics investigation of CAZyme containing genes. Expression profiling across six tissues in E. grandis showed that of the 2,542 CAZyme-domain containing genes in the $E$. grandis 
genome, $80.5 \%(2,044)$ are expressed in at least one tissue (Additional file 8 ). The relative proportions of transcript abundance for each CAZyme domain class were similar across tissues (Figure 2), although the expression of $\mathrm{GH}$ and GT domain classes were proportionally higher in the immature xylem. GTs constitute $44.5 \%$ of expression investment of CAZyme domain containing genes in the immature xylem vs. $35.9 \%$ in the young leaf. GHs account for $39.8 \%$ of the transcript abundance of CAZyme domain containing genes in the immature xylem, and $29.7 \%$ in the young leaf (Figure 2). CE domain family expression was proportionally lower in the phloem and immature xylem compared to the young leaf, mature leaf, flowers and shoot tips, making up 7\% of the total CAZyme expression investment in the immature xylem and $20.3 \%$ in the young leaf. Variation at the level of individual CAZyme domain families was observed, and is discussed below.

The majority of GT domain families showed fairly low levels of ubiquitous expression across six tissues in E. grandis tissues (Figure 3, Additional file 9). We identified domain families that had expression levels higher in a single tissue compared to the remaining five. Of the forty-seven GT domain families present in the E. grandis genome, nine GT families (GT1, GT2, GT4, GT8, GT14, GT31, GT41, GT43, and GT47)(Figure 3) accounted for $80 \%$ of GT expression in immature xylem (Additional file 9). GT41 had the highest expression investment across all tissues (Figure 3). GT41 proteins often contain repeats of the GT41 domain, and in
E. grandis the gene (Eucgr.L00641) contains seven GT41 repeats and had the highest expression of all GT41 containing proteins at $>6$ million FPKM in the xylem (Additional file 8). The GT41 domain occurs 241 times in the E. grandis genome, of which 120 genes containing this domain are expressed in at least one tissue. In comparison, GT1 occurs 511 times in the genome, of which 332 were expressed in at least one tissue and had lower expression investment in the immature xylem compared to the other five tissues analyzed. Thus, GT1 domains are more prevalent in the genome, and more genes containing this domain were expressed, but the magnitude of expression of these genes was considerably lower than the less abundant GT41 domain containing genes.

GH domain family expression investment across six tissues in E. grandis (Additional file 10: Figure S4, Additional file 9) showed that three GH domain families (GH9, GH16 and GH19) showed relatively high levels of expression in different tissues. GH16 domain containing genes were highly expressed in the immature xylem and phloem, and GH19 domain containing genes were highly expressed in the immature xylem and shoot tips. The GH16 domain family is present in the xyloglucan endotransglycosylase/ transferase $(\mathrm{XTH})$ gene family, which contribute to side chain hydrolysis or side chain re-arrangement without hydrolysis (Eklöf et al., 2013). GH9 domain families were also preferentially expressed in the immature xylem compared to the other tissues, the overall higher expression investment in the immature xylem being due to fewer

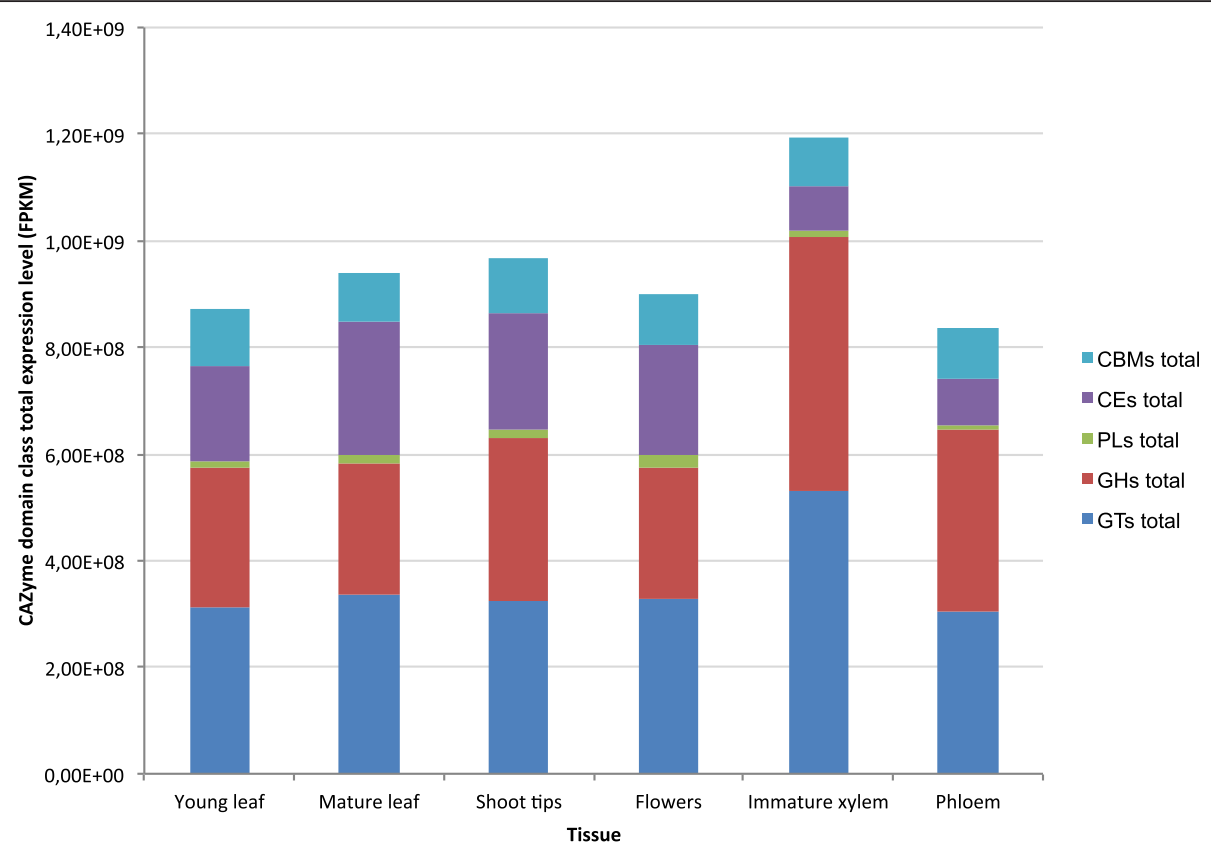

Figure 2 Total gene expression levels of five CAZyme domain classes across six tissues in E. grandis. The $y$-axis represents the mRNA-Seq expression data in FPKM, and the x-axis the tissue type analyzed. The average expression data in FPKM for each gene can be found in Additional file 2. 


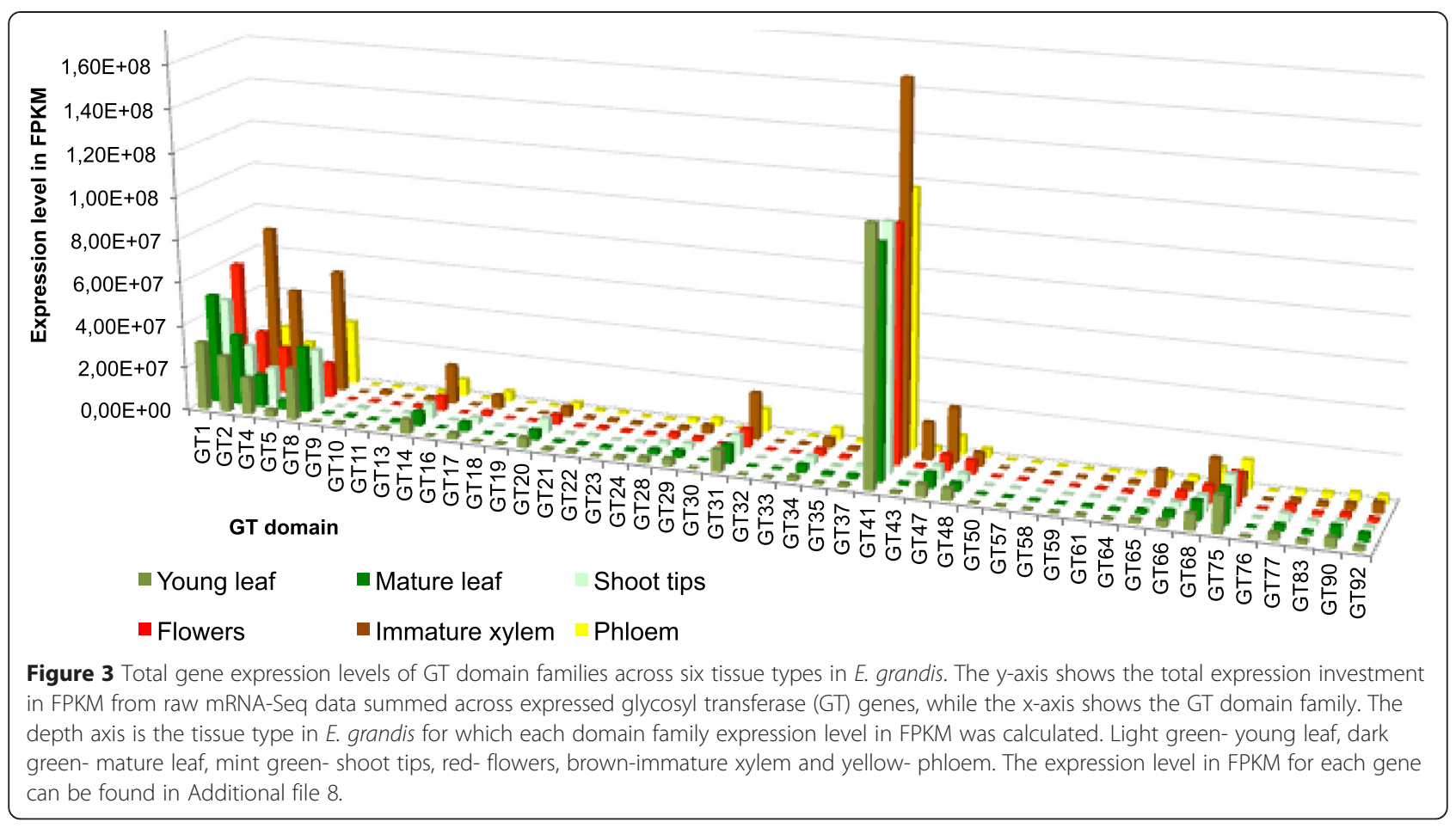

genes (18) being expressed at higher levels than in the other tissues, similar to the GT41 domain containing genes (Additional file 10: Figure S4, Additional file 9). The most highly expressed CAZyme gene in E. grandis xylem was a GH19 family gene, Eucgr.H04034 at 1,01E + 08 FPKM (Additional file 8). The $A$. thaliana ortholog AT3G16920 is a chitinase-like (CTL2) gene, which is known to be involved in cellulose synthesis [49].

PL families have relatively few CAZyme domain families (13) across all species, including the four expressed in $E$. grandis. The expression investment of PL domain families across six tissues in E. grandis showed that all four PL domain families are expressed at diverse levels in all tissues (Additional file 11: Figure S5). PL1 and PL10 showed high expression investment in the flowers compared to the other five tissues. There were no PL families that showed high expression in woody tissues compared to non-woody tissues. CEs showed interesting expression investment across six tissues in E. grandis in that they were expressed fairly ubiquitously at the same level across all tissues (Additional file 12: Figure S6), leading to their lower proportional expression investment level in the immature xylem compared to GT and GH expression investment. Of the $12 \mathrm{CE}$ domain families that were expressed in the $E$. grandis genome, most were expressed at the same level across the different tissues. The exception to this pattern is CE16, which had low relative expression in the immature xylem and phloem, despite having the highest level of expression across the remaining four tissues of all the $\mathrm{CE}$ families. CE16 domain-containing genes are acetyl xylan esterases which de-acetylate preferentially at the $O-3$ and O-4 positions of the backbone xylopyranosyl residues [50].

Most CBM domain families did not show preferential expression investment, and were expressed at the same (relative) level in three or more tissues (Additional file 13: Figure S7). There are two exceptions: CBM18 (chitin-binding), which is highly expressed in young leaf and shoot tips compared to the other four tissues, and CBM22 (xylan binding), which is highly expressed in the immature xylem compared to the other five tissues. CBM57, which is also the most abundant CBM in both E. grandis and P. trichocarpa, showed the highest expression investment of all the CBM domains expressed in the mature leaf, immature xylem and the shoot tips, while CBM43 has the highest expression investment in the flowers and phloem. CBM43 and CBM57 together contributed $51 \%$ of the total average expression investment out of $17 \mathrm{CBM}$ domain families in all tissues. CBM57 was first described in malectin [51], and is involved in the recognition of Glc2-N-glycans. In $E$. grandis, all of the proteins containing these domains are kinases, including mainly LRR protein kinases. Some of the ones highly expressed in wood include HERK1 and THESEUS (brassinosteroids responsive and required for cell elongation during vegetative growth) $[52,53]$.

\section{Comparative expression investment of CAZyme domains} in $E$. grandis and $P$. trichocarpa

To assess the expression investment of CAZyme domain families in two distantly related angiosperm tree species, 
we compared the transcript abundance of CAZyme domain families in E. grandis and P. trichocarpa xylem and leaf tissue (Additional file 9). The pattern of CAZyme family expression was considered to be the same if the family was expressed higher in the xylem than in the leaf in both species and vice versa. The absolute transcript abundance in FPKM cannot be directly compared between these analyses as the experiments were conducted independently, with gene length $(\mathrm{K})$ and sequence depth (M) parameters normalized within the individual transcriptomes for each species. Relative expression values in each tissue can however be compared to identify common expression patterns between the two species.

For the GT family of CAZyme genes, the expression pattern was similar in E. grandis and P. trichocarpa. The majority of GT domain families were expressed at a low level in E. grandis and P. trichocarpa xylem and leaf tissue, which indicates that they are involved in other aspects of carbohydrate metabolism, rather than $\mathrm{CW}$ biosynthesis (Figure 4). GT1 family showed higher expression investment in the leaf tissue as opposed to the xylem tissue in both $E$. grandis and $P$. trichocarpa. The
GT domain families identified in this study as contributing to the majority $(80 \%)$ of expression investment in the immature xylem compared to the other five tissues in E. grandis (Figure 3, Additional file 9) showed greater expression investment in both $E$. grandis and P. trichocarpa xylem compared to leaf (Figure 4). These include the domain families that have been implicated in cellulose and hemicellulose biosynthesis, namely GT2, GT4, GT8, GT14, GT31, GT43, and GT47. The conservation of these expression investment patterns between source (mature leaves) and sink (immature xylem) tissues of divergent tree species indicates a conserved mechanism for CW biosynthesis at the functional domain level, and highlights the importance of regulation of these genes at the level of transcript abundance.

The expression pattern of GH domains in E. grandis and $P$. trichocarpa was similar for most of the GH domain families (Additional file 14: Figure S8a, b). GH4 family was not expressed in the E. grandis tissues studied, including the xylem, while it was expressed at relatively low levels in the xylem and leaf tissue of $P$. trichocarpa. Furthermore, GH57, 62 and 80 were not

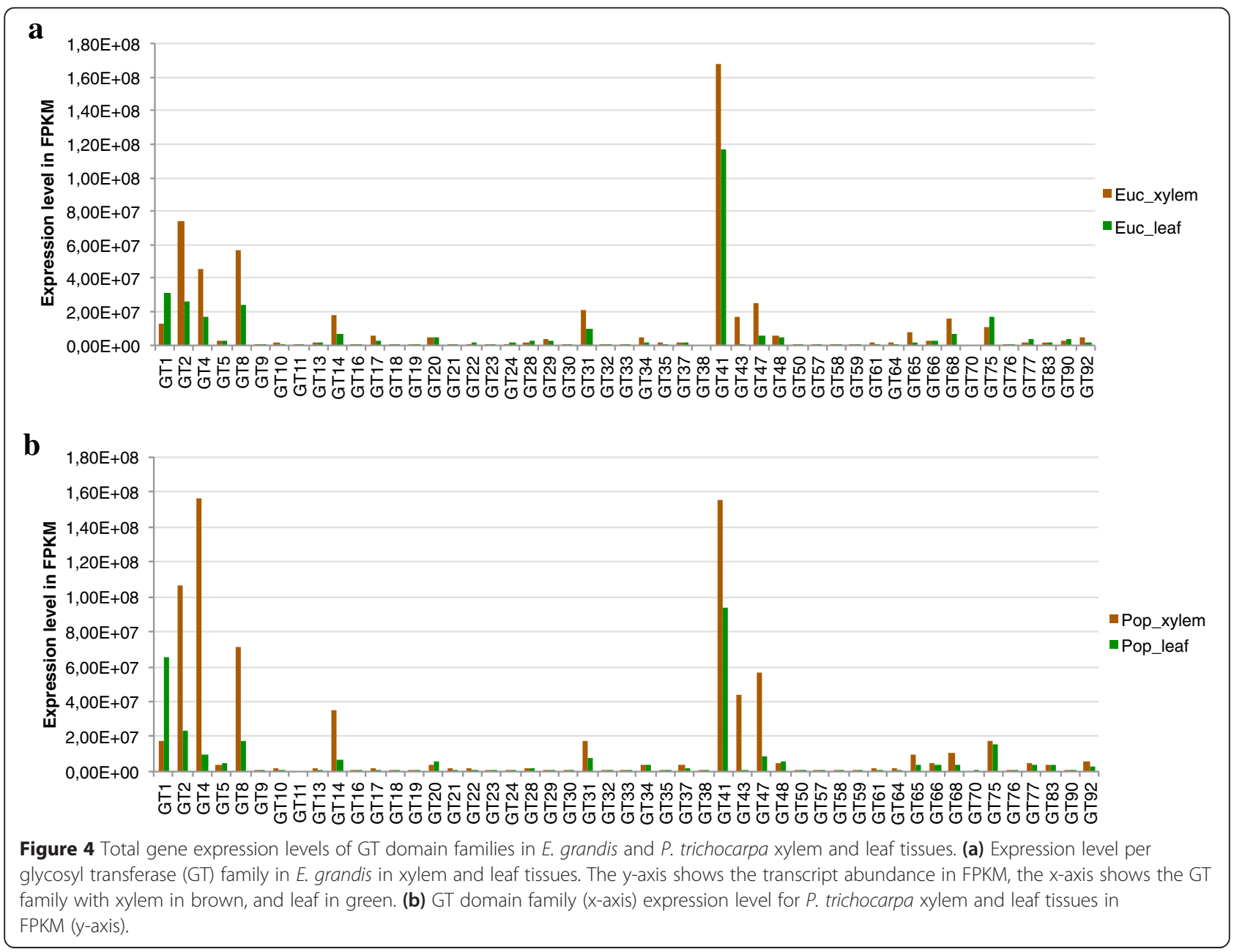


expressed in $P$. trichocarpa xylem and leaf, while they were expressed at low levels in E. grandis. GHs that were expressed at low levels in one species and not in the other may be involved in specific defense or response to abiotic factors, and were thus not captured in a tissue transcriptome of either species. As with E. grandis, the most highly expressed CAZyme in the immature xylem of P. trichocarpa was a CTL2 (GH19 containing) homolog, Potri.010G141600 (Additional file 8).

Comparison of PL family expression between $E$. grandis and $P$. trichocarpa xylem and leaf tissue (Additional file 15: Figure S9a, b), showed that PL1 had a higher expression investment in the xylem as compared to the leaf in both species, with xylem:leaf ratios of 1.9 and 7.1 in $E$. grandis and $P$. trichocarpa, respectively. The same four PL domain families are present in the genome and expressed in both E. grandis and P. trichocarpa. The CE domain class showed variable expression investment patterns between xylem and leaf tissues for $E$. grandis and $P$. trichocarpa (Additional file 16: Figure S10a, b). CE2, 3 and 5 were not expressed in E. grandis and were expressed at relatively low levels in $P$. trichocarpa xylem and leaf. CE15 was expressed in E. grandis and not in $P$. trichocarpa. The CE8 domain family contains pectin methylesterase genes (Jolie et al., 2010), and was more highly expressed in P. trichocarpa xylem than in the leaf, while E. grandis showed the opposite trend (Additional file 16: Figure S10a, b). CE16 had the highest expression investment in the leaf tissue of all the CE domain families expressed in both species. CBMs showed different expression investment patterns between $P$. trichocarpa and $E$. grandis xylem and leaf tissues in a number of families (Additional file 17: Figure S11a, b). Of all the CBMs expressed in E. grandis xylem and leaf, CBM18 showed the highest expression investment in the leaf, while CBM57 had the highest expression level in xylem. In $P$. trichocarpa, of all the CBM domain families expressed, CBM47 had the highest expression in both xylem and leaf (Additional file 17: Figure S11a, b).

\section{Discussion}

Our analysis revealed that the genomic content of CAZyme domains in evolutionary diverse plant genomes is conserved with respect to the ratios of CAZyme classes, although the absolute frequencies vary (Figure 1). Our hypothesis, based on previous findings [30], was that woody perennials would possess a larger proportion of GTs for aspects of carbohydrate metabolism needed to support a large commitment to secondary wall biosynthesis during extensive secondary xylem development (wood production). We found that, in fact, all land plants analyzed show a genomic ratio of 4:3 of GTs to GHs, regardless of their relative investment in different types of carbohydrate metabolism. When considering the maintenance of the ratios of the different CAZyme classes within the genomes between plant species, we observe that the ratio of functional enzymatic domains is maintained, despite differences in whole-genome-, tandem- and segmental duplication events between the species considered. Thus, CAZymes and carbohydrate metabolism may be subject to gene dosage balance selection $[54,55]$. This is also supported by the conservation of relative expression ratios of the majority of CAZyme classes operating in source and sink tissues of Eucalyptus and Populus.

These results, combined with our examination of the presence of unique domains in the genomes of plants, leads us to conclude that the genomic potential to metabolize carbohydrates for wood production is not associated with the emergence of unique CAZyme domain famies. The fact that $P$. patens, despite relatively low numbers of CAZyme genes, has a larger genomic diversity of CAZyme domain families than almost all vascular plant species considered, indicates that primary cell wall (PCW) and SCW metabolism employs a standard set of CAZyme domains in different tissue types of different land plant species. Within woody species, the diversity of CAZyme domain families may contribute to wood formation via unique combinations and regulatory mechanisms of ancestral domains within the genomic and transcriptomic context. We have found that combinations of CAZyme domains do not differentiate woody plants from non-woody plants, as the majority of the types of domain combinations in complex proteins are common between lineages, with low promiscuity of domains (Additional file 7).

Using a comparative transcriptomics approach we were able to define a global view of carbohydrate metabolism in carbon source and sink tissues, and the conservation of transcriptional regulation of CAZyme domains between the divergent woody trees Eucalyptus and Populus. The most highly expressed genes in both $E$. grandis immature xylem and $P$. trichocarpa xylem were the GH19 domain-containing genes Eucgr.H04034 and Potri.010G141600, respectively. CTL2; along with its homolog CTL1, modifies cellulose microfibrils as they are extruded from the CesA complex, as illustrated by the reduced levels of crystalline cellulose in double knockdown mutants of $c t l 1 / c t l 2$ [49]. This, as well as the high and preferential expression of GH16 and GH9 containing proteins (e.g. homologs of KOR1 and GH9B7 in both species), highlights the importance of domains responsible for degradation acting as modifiers to the synthesis of SCW cellulose.

GTs known to synthesize cellulose and hemicellulose showed greater expression investment in the immature xylem compared to the other tissues in E. grandis (Figure 3). Furthermore, these GT domain families 
displayed conserved expression patterns in E. grandis and $P$. trichocarpa xylem and leaf (Figure 4), indicating the importance of relative stoichiometric conservation of functional carbohydrate enzymatic processes at the transcript level. This pattern of conserved domain expression investment in xylem is seen in GT2, GT4, GT8, GT14, GT31, GT41, GT43, and GT47domain families. GT41 family genes are GlcNAc transferases, involved in a multitude of functions, predominantly intracellular signaling [47]. Signaling provides the sensitive feedback necessary to coordinate the deposition of $\mathrm{CW}$ polysaccharides. GT41-mediated modification of proteins can be compared to phosphorylation, as it is a dynamic method of post-translational modification for cytoplasmic and nuclear proteins. GT41 domain containing proteins are also the most complex CAZymes, with greater than four GT41 domain repeats within a single gene found across all plant species analyzed.

GT43 family members IRX9 and IRX14) [56] and GT47 family members (e.g. FRAGILE FIBER8) [57] are known to be involved in xylan biosynthesis. GT43 gene family members are responsible for xylan backbone biosynthesis and have conserved biochemical functions across vascular plants [56]. GT8 domain family containing genes showed high expression investment in the immature xylem compared to the other tissues analyzed, and members of this gene family have been characterized as xylan glucuronyl transferases, including the $P A R$ VUS, GALACTURONOSYLTRANSFERASE, and GALAC TURONOSYLTRANSFERASE-LIKE (GAUT/GATLI) genes [58,59]. The GT31 domain containing gene At4g21060 in A. thaliana has been shown to encode a galactosyltransferase that is responsible for arabinogalactan protein galactosylation during backbone formation [60]. Amongst the other GTs that displayed preferential expression in immature xylem than the other tissues, we found GT65 and GT68, which are fucosyl and oligosaccharide transferases respectively [13]. GT and GT-like enzymes accounted for $20 \%$ of the proteome of $A$. thaliana Golgi apparatus, including GT14, GT8 and GT31 domain containing proteins [61], lending some confidence to the expression abundance observed in this study.

\section{Conclusions}

A key finding of this study is that the CAZyme containing genes in plant genomes have a conserved ratio between species, regardless of their carbohydrate metabolic strategy or the tandem, segmental or documented whole genome duplication events in their evolutionary history. Although we find evidence for lineage specific diversity of CAZyme families in plant genomes, the domain family diversity of CAZymes cannot be used to discriminate the eudicot and monocot lineages, or woody and herbaceous species. The expression pattern of the CAZyme domains responsible for cellulose and xylan biosynthesis appears to be stoichiometrically conserved between Eucalyptus and Populus. This study highlights the importance of transcriptional regulation in the evolution of wood development as opposed to genomic innovations in the enzymatic domains responsible for carbohydrate metabolism.

\section{Methods}

\section{Genome-wide analysis of CAZyme domains in plant} species

All CAZyme domains for twenty-two plant species (Table 1) in Phytozome v8.0 [35] were obtained from the dbCAN database [33,34]. The plant species examined for the genome-wide analysis of CAZyme domains were chosen in order to encompass the Viridiplantae lineage (see Table 1 for all species and abbreviations), including Chlorophyta (including only C. reinhardtii and V. carteri), Embryophyta, encompassing $P$. patens onwards, Tracheophyta, encompassing S. moellendorffii onwards and monocot and dicot representatives of the Magniliophyta.

Analysis was performed using custom Python scripts (Python v2.6, Additional files 18 and 19). The Python language [62] was used in this study to write and execute custom scripts to rapidly, reproducibly and accurately analyze large tables of data. The primary applications of these scripts included basic data manipulation of the text files obtained from the dbCAN database, firstly extracting all the CAZyme domains present in each genome and collating them by domain family (Additional file 18), and secondly, counting all the CAZyme domains in each family per genome (Additional file 19). These collated and counted values of domain frequency per CAZyme domain family per genome were analyzed further in Excel. We classified three parameters, namely i. Frequency- the absolute numbers and relative frequencies of annotated genes within each of the five CAZyme domain classes, and the families assigned to these classes in the genomes of all twenty-two species, ii. Diversity- the number and type of individual CAZyme domain families within and between species, and iii. Complexity- occurrence, frequency and diversity of CAZyme domains within annotated genes. Covariance analysis to determine within and between species CAZyme domain class relative frequency variation was done using SAS v9.3 (Statistical Analysis Software- SAS Institute Inc.).

Diversity of CAZyme domains were analyzed by grouping and counting all the individual domains present in each genome (including each domain in multi-domain proteins) into their families based on $\mathrm{dbCAN}$ annotations. Complexity analysis was performed on a subset of ten species representing major lineages of land plant evolution (Table 1). The analysis of CAZyme 
domain complexity with annotated genes in each genome involved identifying all annotated genes that contained multiple CAZyme domains. Firstly, the number of annotated domains per gene in each of the ten genomes was calculated and visualized in Excel. Secondly, all genes containing multiple annotated CAZyme domains were separated based on whether they consisted solely of repeat CAZyme domains, or contained unique CAZyme domain families. These two categories of multiple CAZyme domain containing annotated genes were then analyzed either by the frequency of the domain repeats, or by the combinations of unique domains they contained, and subsequently compared across species.

\section{Gene expression analysis of CAZyme-coding genes in $E$. grandis and $P$. trichocarpa}

In previous studies, next generation deep mRNAsequencing using the Illumina platform was used to quantify the genome-wide expression in the transcriptomes of multiple tissues in E. grandis and P. trichocarpa $[37,48,63,64]$. Genome-wide transcriptome data for six tissues in E. grandis from Dr. C. Hefer was obtained for analysis of the transcript abundance of all expressed CAZyme genes [63]. The tissues analyzed in this study were: Young leaves, mature leaves, immature xylem, phloem, shoot tips, and flowers of E. grandis $[48,63]$. mRNA-sequencing data from young leaf and immature xylem tissue of $P$. trichocarpa was used for comparison to the E. grandis transcriptome [64]. The expression levels of every gene in each tissue/organ were averaged across three biological replicates, and filtered for genes containing CAZyme domains in E. grandis and P. trichocarpa from the dbCAN database [34] for further analysis.

The transcript abundance of genes from mRNA-Seq can be quantified as Fragments Per Kilobase of exon per Million fragments mapped (FPKM) [65]. FPKM parameters $\mathrm{K}$ and $\mathrm{M}$ are optimized to individual experiments in the software used to assemble the transcriptomes, in this case Cufflinks [66], was used (for more detail, refer to [64]). To infer the investment of expression of CAZyme domain families in each tissue, the total transcript abundance for all genes in each CAZyme domain family was summed, and compared that total to the FPKM expression investment values for the other tissues, using Excel for numerical comparisons and visualization. When calculating total expression investment of domain families, genes annotated with multiple CAZyme domain families were treated differently: If the gene was annotated as consisting solely of repeats of the same CAZyme domain, the total transcript abundance of the entire gene was added once to the CAZyme domain family total transcript abundance. Therefore repeat domains of the same CAZyme family were ignored when calculating CAZyme domain family specific transcript abundance. If the gene was annotated as having multiple domains from different CAZyme domain families, the transcript abundance of that gene was added separately to each domain family once. For example, a gene annotated as having domains "X-X-Y", would have the FPKM value of the gene added once to "family $\mathrm{X}$ expression investment total", and once to "family Y expression investment total".

\section{Additional files}

Additional file 1: Table S1. Relative standard deviation (RSD) (absolute co-efficient of variation) between plant species.

Additional file 2: Excel file: CAZyme domain family frequency across twenty-two plant species.

Additional file 3: Figure S1. Domain family frequency distribution across twenty-two species.

Additional file 4: Figure S2. Number of CAZy domains in complex CAZy domain containing proteins across ten representative plant species. Additional file 5: Excel file: CAZyme domain containing protein complexity summary in 10 plant species.

Additional file 6: Figure S3. Venn diagram of CAZyme domain unique combinations within complex proteins in five eudicots.

Additional file 7: Excel file: Frequency of unique CAZyme domain combinations in complex proteins in 10 plant species (in separate tabs).

Additional file 8: Excel file: Expressed CAZyme domain containing proteins (FPKM) and domain content in E. grandis.

Additional file 9: Excel file: CAZyme domain family expression in FPKM with standard deviation in E. grandis.

Additional file 10: Figure S4. GH domain family expression levels across six tissues in E. grandis in FPKM.

Additional file 11: Figure S5. PL domain family expression levels across six tissues in E. grandis in FPKM.

Additional file 12: Figure S6. CE domain family expression level across six tissues in E. grandis in FPKM.

Additional file 13: Figure S7. CBM domain family expression level across six tissues in E. grandis in FPKM.

Additional file 14: Figure S8. Comparative expression patterns of $\mathrm{GH}$ domain families in E. grandis and P. trichocarpa.

Additional file 15: Figure S9. Comparative expression patterns of PL domain families in E. grandis and P. trichocarpa.

Additional file 16: Figure S10. Comparative expression patterns of $C E$ domain families in E. grandis and P. trichocarpa.

Additional file 17: Figure S11. Comparative expression patterns of CBM domain families in E. grandis and P. trichocarpa.

Additional file 18: Python script domain_counter.py: Used to count the frequency of multiple domains in all species for all families across columns. Comments included in file.

Additional file 19: Python script domain_pull.py: Used to sort gene frequency based on domain family. Comments included in file.

\section{Abbreviations}

CAZymes: Carbohydrate Active enZymes; GT: Glycosyl transferase;

GH: Glycosyl hydrolase; CE: Carbohydrate esterase; PL: Polysaccharide lyase; CBM: Carbohydrate binding module; CW: Cell wall; SCW: Secondary cell wall; FPKM: Fragments Per Kilobase of exon per Million fragments mapped; XTH: Xyloglucan endotransglycosylase/transferase; CESA: Cellulose synthase A.

Competing interests

The authors declare that they have no competing interests. 


\section{Authors' contributions}

The study was conceived by AAM and EM, and designed by DP, EM, SDM and AAM. AAM, CJD and SDM generated the transcriptome data, which was processed by $\mathrm{CAH}$ and FJ. DP and AK did the data analysis. DP, EM, SDM, and AAM were involved in data interpretation. The manuscript was drafted by DP, manuscript editing and revisions were performed by all listed authors. All authors have read and approved the final version of the manuscript.

\section{Acknowledgements}

This work was supported through the Forest Molecular Genetics Programme by Sappi, the Technology and Human Resources for Industry Programme (THRIP, UID 80118), the National Research Foundation (NRF, UID18312 and 86936) and the Department of Science and Technology (DST) of South Africa. The authors thank Andrew dos Santos (University of Pretoria) for his technical assistance in contribution to the study.

\section{Author details}

${ }^{1}$ Department of Genetics, Forestry and Agricultural Biotechnology Institute (FABI), University of Pretoria, Private bag X20 Hatfield, Pretoria 0028, South Africa. ${ }^{2}$ Centre for Bioinformatics and Computational Biology, Genomics Research Institute (GRI), University of Pretoria, Private bag X20 Hatfield, Pretoria 0028, South Africa. ${ }^{3}$ Evolutionary Bioinformatics Group, Institute for Evolution and Biodiversity, Hufferstr. 1, Munster D48149, Germany. ${ }^{4}$ Department of Botany, University of British Columbia, 6270 University Blvd, Vancouver, BC V6T 1Z4, Canada. ${ }^{5}$ Department of Wood Science, University of British Columbia, 2424 Main Mall, Vancouver, BC V6T 1Z4, Canada.

\section{Received: 17 October 2014 Accepted: 23 April 2015}

\section{Published online: 22 May 2015}

\section{References}

1. Coutinho PM, Stam M, Blanc E, Henrissat B. Why are there so many carbohydrate-active enzyme-related genes in plants ? Trends Plant Sci. 2003;8:563-5.

2. Wilson IBH. Glycosylation of proteins in plants and invertebrates. Curr Opin Struct Biol. 2002;12:569-77.

3. Grattapaglia D, Plomion C, Kirst M, Sederoff RR. Genomics of growth traits in forest trees. Curr Opin Plant Biol. 2009;12:148-56.

4. Hinchee M, Rottmann W, Mullinax L, Zhang C, Chang S, Cunningham M, et al. Short-rotation woody crops for bioenergy and biofuels applications. Vitr Cell Dev Biol - Plant. 2009;45:619-29.

5. Plomion C, Stokes A, Leprovost G. Wood formation in trees. Plant Physiol. 2001;127(December):1513-23

6. Cosgrove DJ. Growth of the plant cell wall. Nature. 2005;6:850-61.

7. Hansen SF, Bettler E, Rinnan A, Engelsen SB, Breton C. Exploring genomes for glycosyltransferases. Mol Biosyst. 2010;6:1773-81.

8. Cantarel BL, Coutinho PM, Rancurel C, Bernard T, Lombard V, Henrissat B. The Carbohydrate-Active EnZymes database (CAZy): an expert resource for glycogenomics. Nucleic Acids Res. 2009;37(Database issue):233-8.

9. Henrissat B, Davies GJ. Glycoside hydrolases and glycosyltransferases. Families, modules, and implications for genomics. Plant Physiol. 2000;124(December):1515-9.

10. Henrissat B, Davies G. Structural and sequence-based classification of glycoside hydrolases. Carbohydrates and glycoconjugates. 1997;7:637-44.

11. Henrissat B, Coutinho PM, Davies GJ. A census of carbohydrate-active enzymes in the genome of Arabidopsis thaliana. Plant Mol Biol. 2001:47:55-72.

12. Van Tilbeurgh $H$, Tomme $P$, Claeyssens M, Bhikhabhai R, Pettersson G. Limited proteolysis of the cellobiohydrolase I from Trichoderma reesei. FEBS Lett. 1986;204:223-7.

13. The Carbohydrate-Active enZYmes Database. www.cazy.org. Accessed 3 June 2014

14. Lairson LL, Henrissat B, Davies GJ, Withers SG. Glycosyltransferases: structures, functions, and mechanisms. Annu Rev Biochem. 2008;77:521-55.

15. Dhugga KS. Building the wall: genes and enzyme complexes for polysaccharide synthases. Curr Opin Plant Biol. 2001;4:488-93.

16. Djerbi S, Aspeborg H, Schrader J, Coutinho PM, Stam M, Nilsson P, et al. Carbohydrate-active enzymes involved in the secondary cell wall biogenesis in hybrid Aspen. Plant Physiol. 2005;137(March):983-97.
17. Lee C, Teng Q, Huang W, Zhong R, Ye Z. The Poplar GT8E and GT8F glycosyltransferases are functional orthologs of Arabidopsis PARVUS involved in glucuronoxylan biosynthesis. Plant Cell Physiol. 2009;50:1982-7.

18. Li Q, Min D, Wang JP-Y, Peszlen I, Horvath L, Horvath B, et al. Down-regulation of glycosyltransferase 8D genes in Populus trichocarpa caused reduced mechanical strength and xylan content in wood. Tree Physiol. 2011;31:226-36.

19. Serapiglia MJ, Cameron KD, Stipanovic AJ, Smart LB. Correlations of expression of cell wall biosynthesis genes with variation in biomass composition in shrub willow (Salix spp.) biomass crops. Tree Genet Genomes. 2011;10:1-9.

20. Chiniquy D, Sharma V, Schultink A, Baidoo EE, Rautengarten C, Cheng K. XAX1 from glycosyltransferase family 61 mediates xylosyltransfer to rice xylan. Proc Natl Acad Sci U S A. 2012;109:17117-22.

21. Dhugga KS. Biosynthesis of non-cellulosic polysaccharides of plant cell walls. Phytochemistry. 2012;74:8-19.

22. Minic Z. Physiological roles of plant glycoside hydrolases. Planta. 2008;227:723-40.

23. Linhardt RJ, Galliher PM, Cooney CL. Polysaccharide lyases. Appl Biochem Biotechnol. 1986;12:135-76.

24. Garron M-L, Cygler M. Structural and mechanistic classification of uronic acid-containing polysaccharide lyases. Glycobiology. 2010;20:1547-73.

25. Cantarel BL, Lombard V, Henrissat B. Complex carbohydrate utilization by the healthy human microbiome. PLoS One. 2012;7:e28742.

26. Tsai AY-L, Canam T, Gorzsás A, Mellerowicz EJ, Campbell MM, Master ER. Constitutive expression of a fungal glucuronoyl esterase in Arabidopsis reveals altered cell wall composition and structure. Plant Biotechnol J. 2012;10:1077-87.

27. Boraston AB, Bolam DN, Gilbert HJ, Davies GJ. Carbohydrate-binding modules: fine-tuning polysaccharide recognition. Biochemistry. 2004;382:769-81.

28. Hervé C, Rogowski A, Blake AW, Marcus SE, Gilbert HJ, Knox JP. Carbohydrate-binding modules promote the enzymatic deconstruction of intact plant cell walls by targeting and proximity effects. Proc Natl Acad Sci U S A. 2010;107:15293-8.

29. Levy I, Shoseyov O. Cellulose-binding domains: Biotechnological applications. Biotechnol Adv. 2002;20:191-213.

30. Geisler-Lee J, Geisler M, Coutinho PM, Segerman B, Nishikubo N, Takahashi J, et al. Poplar carbohydrate-active enzymes. Gene identification and expression analyses. Plant Physiol. 2006;140(March):946-62.

31. Nasir A, Naeem A, Jawad Khan M, Lopez-NicoraArshan HD, Caetano-Anollés G. Annotation of protein domains reveals remarkable conservation in the functional make up of proteomes across Superkingdoms. Gene. 2011;2:869-911.

32. Kersting AR, Bornberg-Bauer E, Moore AD, Grath S. Dynamics and adaptive benefits of protein domain emergence and arrangements during plant genome evolution. Genome Evol Biol. 2012:4:316-29.

33. Yin Y, Mao X, Yang J, Chen X, Mao F. Xu Y: dbCAN: a web resource for automated carbohydrate-active enzyme annotation. Nucleic Acids Res. 2012;479:1-7.

34. dbCAN: DataBase for automated Carbohydrate-active enzyme ANnotation http://csbl.bmb.uga.edu/dbCAN/. Accessed 30 March 2014

35. Phytozome .http://phytozome.jgi.doe.gov. Accessed 5 February 2015

36. Groover AT. What genes make a tree a tree? Trends Plant Sci. 2005;10:210-4.

37. Myburg AA, Grattapaglia D, Tuskan GA, Hellsten U, Hayes RD, Grimwood J, et al. The genome of Eucalyptus grandis. Nature. 2014;510:356-62.

38. Freeling M. Bias in plant gene content following different sorts of duplication: tandem, whole-genome, segmental, or by transposition. Annu Rev Plant Biol. 2009;60:433-53.

39. Proost $S$, Pattyn P, Gerats T, Van de Peer Y. Journey through the past: 150 million years of plant genome evolution. Plant J. 2011;66:58-65.

40. Vanneste K, Baele G, Maere S, Van de Peer Y. Analysis of 41 plant genomes supports a wave of successful genome duplications in association with the Cretaceous-Paleogene boundary. Genome Res. 2014;113:1-37.

41. Maere S, De Bodt S, Raes J, Casneuf T, Van Montagu M, Kuiper M, et al. Modeling gene and genome duplications in eukaryotes. Proc Natl Acad Sci U S A. 2005;102:5454-9.

42. Mewalal R, Mizrachi E, Mansfield SD, Myburg AA. Cell wall-related proteins of unknown function: Missing links in plant cell wall development. Plant Cell Physiol. 2014;50:1-13.

43. Nystedt B, Street NR, Wetterbom A, Zuccolo A, Lin Y-C, Scofield DG, et al. The Norway spruce genome sequence and conifer genome evolution. Nature. 2013;497:579-84.

44. ConGenIE .http://congenie.org/. Accessed 12 May 2014.

45. Tordai H, Nagy A, Farkas K, Bányai L, Patthy L. Modules, multidomain proteins and organismic complexity. FEBS J. 2005;272:5064-78. 
46. Martinez-Fleites C, He Y, Davies GJ. Structural analyses of enzymes involved in the O-GlcNAc modification. Biochim Biophys Acta. 1800;2010:122-33.

47. Breton C, Fournel-Gigleux S, Palcic MM. Recent structures, evolution and mechanisms of glycosyltransferases. Curr Opin Struct Biol. 2012;22:540-9.

48. Hefer C, Mizrachi E, Joubert F, Myburg A. The Eucalyptus genome integrative explorer (EucGenIE): a resource for Eucalyptus genomics and transcriptomics. BMC Proc. 2011;5 Suppl 7:1.

49. Sánchez-Rodríguez C, Bauer S, Hématy K, Saxe F, Ibáñez AB, Vodermaier V, et al. Chitinase-like1/pom-pom1 and its homolog CTL2 are glucan-interacting proteins important for cellulose biosynthesis in Arabidopsis. Plant Cell. 2012;24:589-607.

50. Pawar PM-A, Koutaniemi S, Tenkanen M, Mellerowicz EJ. Acetylation of woody lignocellulose: significance and regulation. Front Plant Sci. 2013;4(May):118-26.

51. Schallus T, Jaeckh C, Fehér K, Palma AS, Liu Y, Simpson JC, et al. Malectin: A novel carbohydrate-binding protein of the endoplasmic reticulum and a candidate player in the early steps of protein N-glycosylation. Mol Biol Cell. 2008;19(August):3404-14.

52. Guo H, Li L, Ye H, Yu X, Algreen A, Yin Y. Three related receptor-like kinases are required for optimal cell elongation in Arabidopsis thaliana. Proc Natl Acad Sci U S A. 2009;106:7648-53

53. Hématy K, Sado P-E, Van Tuinen A, Rochange S, Desnos T, Balzergue S, et al. A receptor-like kinase mediates the response of Arabidopsis cells to the inhibition of cellulose synthesis. Curr Biol. 2007;17:922-31.

54. Veitia RA, Bottani S, Birchler JA. Cellular reactions to gene dosage imbalance: genomic, transcriptomic and proteomic effects. Trends Genet. 2008;24:390-7

55. De Smet $R$, Van de Peer $Y$. Redundancy and rewiring of genetic networks following genome-wide duplication events. Curr Opin Plant Biol. 2012;15:168-76.

56. Lee C, Zhong R, Ye Z-H. Arabidopsis family GT43 members are xylan xylosyltransferases required for the elongation of the xylan backbone. Plant Cell Physiol. 2012;53:135-43.

57. Doering A, Lathe R, Persson S. An update on xylan synthesis. Mol Plant. 2012:5:769-71.

58. Yin $Y$, Chen $H$, Hahn MG, Mohnen D, Xu Y. Evolution and function of the plant cell wall synthesis-related glycosyltransferase family 8. Plant Physiol. 2010;153:1729-46.

59. Rennie EA, Hansen SF, Baidoo EEK, Hadi MZ, Keasling JD, Scheller HV. Three members of the Arabidopsis glycosyltransferase family Are xylan glucuronosyltransferases. Plant Physiol. 2012;159(August):1408-17.

60. Basu D, Liang Y, Liu X, Himmeldirk K, Faik A, Kieliszewski M, Held M, Showalter AM. Functional identification of a hydroxyproline-O-galactosyltransferase specific for arabinogalactan protein biosynthesis in Arabidopsis. J Biol Chem 2013;288:1-25.

61. Parsons HT, Christiansen K, Knierim B, Carroll A, Ito J, Batth TS, et al. Isolation and proteomic characterization of the Arabidopsis Golgi defines functional and novel components involved in plant cell wall biosynthesis. Plant Physiol. 2012;159:12-26.

62. Lutz M. Learning Python. 3rd Editio. Sebastopol, CA, USA: O'Reilly Meidia, Inc.; 2008:3-9.

63. EucGenIE .http://www.eucgenie.org/. Accessed 20 September 2013.

64. Hefer CA, Mizrachi E, Myburg AA, Douglas CJ, Mansfield SD: Comparative interrogation of the developing xylem transcriptomes of two wood-forming species: Populus trichocarpa and Eucalyptus grandis. New Phytol 2015, doi: 10.11

65. Trapnell C, Salzberg SL. How to map billions of short reads onto genomes. Nat Biotech. 2009:27:455-7.

66. Cufflinks. http://cufflinks.cbcb.umd.edu/. Accessed 17 March 2013.

67. Prochnik SE, Umen J, Nedelcu AM, Hallmann A, Miller SM, Nishii I, et al. Genomic analysis of organismal complexity in the multicellular green alga Volvox carteri. Science (80- ). 2010;329:223-6.

68. Merchant SS, Prochnik SE, Vallon O, Harris EH, Karpowicz SJ, Witman GB, et al. The Chlamydomonas genome reveals the evolution of key animal and plant functions. Science (80- ). 2007;318:245-50.

69. Rensing SA, Lang D, Zimmer AD, Terry A, Salamov A, Shapiro $H$, et al. The Physcomitrella genome reveals evolutionary insights into the conquest of land by plants. Science. 2008:319:64-9.

70. Banks JA, Nishiyama T, Hasebe M, Bowman JL, Gribskov M, DePamphilis C, et al. The Selaginella genome identifies genetic changes associated with the evolution of vascular plants. Science (80- ). 2011;332:960-3.
71. The International Brachypodium Initiative. Genome sequencing and analysis of the model grass Brachypodium distachyon. Nature. 2010;463:763-8.

72. Goff SA, Ricke D, Lan T-H, Presting G, Wang R, Dunn M, et al. A draft sequence of the rice genome (Oryza sativa L. ssp. japonica). Science (80- ). 2002;296:92-100.

73. Schnable PS, Ware D, Fulton RS, Stein JC, Wei F, Pasternak S, et al. The B73 maize genome: complexity, diversity, and dynamics. Science (80-). 2009;326:1112-5.

74. Paterson AH, Bowers JE, Bruggmann R, Dubchak I, Grimwood J, Gundlach H, et al. The Sorghum bicolor genome and the diversification of grasses. Nature. 2009;457:551-6.

75. Aquilegia coerulea Genome Sequencing Project. http://phytozome.jgi.doe.gov. Accessed 14 June 2012

76. Hellsten U, Wright KM, Jenkins J, Shu S, Yuan Y, Wessler SR, et al. Fine-scale variation in meiotic recombination in Mimulus inferred from population shotgun sequencing. Proc Natl Acad Sci. 2013;110(48):19478-82.

77. Jaillon O, Aury J-M, Noel B, Policriti A, Clepet C, Casagrande A, et al. The grapevine genome sequence suggests ancestral hexaploidization in major angiosperm phyla. Nature. 2007:449:463-7.

78. Wu GA, Prochnik S, Jenkins J, Salse J, Hellsten U, Murat F, et al. Sequencing of diverse mandarin, pummelo and orange genomes reveals complex history of admixture during citrus domestication. Nat Biotech. 2014;32:656-62

79. Ming R, Hou S, Feng Y, Yu Q, Dionne-Laporte A, Saw JH, et al. The draft genome of the transgenic tropical fruit tree papaya (Carica papaya Linnaeus). Nature. 2008;452:991-6.

80. The Arabidopsis Genome Initiative. Analysis of the genome sequence of the flowering plant Arabidopsis thaliana. Nature. 2000;408:796-815.

81. Verde I, Abbott AG, Scalabrin S, Jung S, Shu S, Marroni F, et al. The high-quality draft genome of peach (Prunus persica) identifies unique patterns of genetic diversity, domestication and genome evolution. Nat Genet. 2013;45:487-94.

82. Huang S, Li R, Zhang Z, Li L, Gu X, Fan W, et al. The genome of the cucumber, Cucumis sativus L. Nat Genet. 2009:41:1275-81.

83. Schmutz J, Cannon SB, Schlueter J, Ma J, Mitros T, Nelson W, et al. Genome sequence of the palaeopolyploid soybean. Nature. 2010;463:178-83.

84. Tuskan GA, Difazio S, Jansson S, Bohlmann J, Grigoriev I, Hellsten U, et al. The genome of black cottonwood, Populus trichocarpa (Torr. \& Gray). Science (80- ). 2006;313:1596-15604.

85. Chan AP, Crabtree J, Zhao Q, Lorenzi H, Orvis J, Puiu D, et al. Draft genome sequence of the oilseed species Ricinus communis. Nat Biotechnol. 2010:28:951-6.

86. Prochnik S, Marri PR, Desany B, Rabinowicz PD, Kodira C, Mohiuddin M, et al. The Cassava genome: Current progress, future directions. Trop Plant Biol. 2012;5:88-94.

\section{Submit your next manuscript to BioMed Central and take full advantage of:}

- Convenient online submission

- Thorough peer review

- No space constraints or color figure charges

- Immediate publication on acceptance

- Inclusion in PubMed, CAS, Scopus and Google Scholar

- Research which is freely available for redistribution 